\title{
AN ANSWER TO VAN MILL'S QUESTION
}

\author{
ER-GUANG YANG ${ }^{\bowtie}$ and WEI-XUE SHI
}

(Received 10 December 2007)

\begin{abstract}
van Mill et al. posed in 'Classes defined by stars and neighborhood assignments', Topology Appl. 154 (2007), 2127-2134 the following question: Is a star-compact space metrizable if it has a $G_{\delta}$-diagonal? In this paper, we give a negative answer to this question.
\end{abstract}

2000 Mathematics subject classification: 54D20, 54E35.

Keywords and phrases: star-compact, metrizability, $G_{\delta}$-diagonal.

\section{Introduction}

Star-compact spaces are natural generalizations of countably compact spaces (strongly 1-star-compact spaces). van Mill et al. gave an example in [4] showing that starcompact spaces are strictly weaker than countably compact spaces. It is known that [2] a countably compact space with a $G_{\delta}$-diagonal is compact metrizable. van Mill et al. posed the following question in [4].

QUESTION 1.1 [4]. Is a star-compact space metrizable if it has a $G_{\delta}$-diagonal?

In this paper, we give a negative answer to the above question.

Throughout, a space will mean a topological space. Let $A$ be a subset of a space $X$ and $\mathcal{U}$ a family of subsets of $X$. The star, $\operatorname{St}(A, \mathcal{U})$, of the set A with respect to $\mathcal{U}$ is the set $\bigcup\{U \in \mathcal{U} \mid U \cap A \neq \emptyset\}$.

Definition 1.2 [3]. Let $\mathcal{P}$ be a class (or a property) of a space $X . X$ is said to be star- $\mathcal{P}$ (or star-determined by $\mathcal{P}$ ) if for any open cover $\mathcal{U}$ of the space $X$, there is a subspace $Y \subset X$ such that $Y \in \mathcal{P}$ and $\operatorname{St}(Y, \mathcal{U})=X$.

By Definition 1.2, a space $X$ is said to be star-compact (respectively, stardetermined by convergent sequences) if, for any open cover $\mathcal{U}$ of the space $X$, there is a compact subspace $K$ (respectively, a convergent sequence $S$ ) of $X$ such that $\operatorname{St}(K, \mathcal{U})=X($ respectively, $\operatorname{St}(S, \mathcal{U})=X)$.

This work is supported by NSFC, project 10571081.

(C) 2009 Australian Mathematical Society 0004-9727/09 \$A2.00+0.00 
Definition 1.3 [1]. A space $X$ is said to be strongly 1-star-compact (respectively, 1-star-compact) if, for any open cover $\mathcal{U}$ of $X$, there exists a finite subset $F \subset X$ (respectively, a finite subfamily $\mathcal{V}$ of $\mathcal{U}$ ) such that $\operatorname{St}(F, \mathcal{U})=X$ (respectively, $\operatorname{St}(\cup \mathcal{V}, \mathcal{U})=X)$.

It was shown that [1] the notions of strongly 1-star-compact and countably compact are equivalent in a $T_{2}$ space.

\section{An example}

In this section, we give an example which presents a negative answer to Question 1.1. The space used here was constructed by van Douwen et al. [1]. It was proved that the space is second countable and 1-star-compact. We show that it is also star-compact and has a $G_{\delta}$-diagonal but it is not metrizable, which answers Question 1.1 negatively.

EXAMPLE 2.1. There exists a second countable star-compact space $X$ which has a $G_{\delta}$-diagonal but $X$ is not metrizable.

Proof. Let $Y=\bigcup\{[0,1] \times\{n\} \mid n<\omega\}$ and $X=Y \cup\{a\}$, where $a \notin Y$. Topologize $X$ as follows: basic neighborhoods of the point $a$ take the form $\{a\} \cup \bigcup\{[0,1) \times\{n\} \mid$ $n>m\}$, where $m \in \omega$; basic neighborhoods of the other points of $X$ are the usual induced metric open neighborhoods. One readily sees that the space $X$ with this topology is $T_{2}$ second countable and the subspace $[0,1] \times\{n\}$ is compact for all $n \in \omega$ [1].

Since the point $a$ and the closed set $\{(1, n) \mid n \in \omega\}$ cannot be separated by open sets, $X$ is not regular, and hence not metrizable.

Now, we show that $X$ is star-compact. Let $\mathcal{U}$ be an open cover of $X$ consisting of basic open sets of $X$. For each $n \in \omega$, there exists $U_{n} \in \mathcal{U}$ such that $(1, n) \in U_{n}$. For each $n \in \omega, U_{n}$ can be represented as $U_{n}=V_{n} \times\{n\}$, where $V_{n}$ is an open neighborhood of 1 in $[0,1]$. Thus $V_{n} \backslash\{1\} \neq \emptyset$. Pick $x_{n} \in V_{n} \backslash\{1\}$ then $\left(x_{n}, n\right) \in U_{n}$ with $\left(x_{n}, n\right) \neq(1, n)$. Put $K_{1}=\left\{\left(x_{n}, n\right) \mid n \in \omega\right\} \cup\{a\}$ then $K_{1}$ is a compact subset of $X$ and $\{(1, n) \mid n \in \omega\} \subset S t\left(K_{1}, \mathcal{U}\right)$. For the point $a$, choose $U_{a} \in \mathcal{U}$ such that $a \in U_{a}$. Then there exists $m \in \omega$ such that $U_{a}=\{a\} \cup \bigcup\{[0,1) \times\{n\} \mid n>m\}$. It is obvious that $U_{a} \subset S t\left(K_{1}, \mathcal{U}\right)$ since $U_{a} \cap K_{1} \neq \emptyset$. Now, set $K_{2}=\bigcup\{[0,1] \times\{n\} \mid n \leq m\}$; then, being a finite union of compact sets, $K_{2}$ is compact. Obviously, $K_{2} \subset \operatorname{St}\left(K_{2}, \mathcal{U}\right)$. Put $K=K_{1} \cup K_{2}$; then $K$ is a compact subset of $X$ and $\operatorname{St}(K, \mathcal{U})=X$, which shows that $X$ is star-compact.

It remains to show that $X$ has a $G_{\delta}$-diagonal. From the construction of the topology of $X$, we see that $X$ has a countable base $\mathcal{B}$ consisting of basic open sets of $X$. Then $\mathcal{B} \times \mathcal{B}$ is a countable base of $X \times X$. It is easy to verify that every basic open set of $X$ can be represented as the countable union of closed subsets of $X$. Thus every member of $\mathcal{B} \times \mathcal{B}$ can be represented as the countable union of closed subsets of $X \times X$. This, together with the fact that $\mathcal{B} \times \mathcal{B}$ is a countable base of $X \times X$, shows that $X \times X$ is perfect. $X$ being $T_{2}$, we conclude that $X$ has a $G_{\delta}$-diagonal.

This completes the proof. 
REMARK 2.2. Spaces star-determined by convergent sequences which are stronger than star-compact spaces are also generalizations of countably compact spaces (strongly 1-star-compact spaces). van Mill et al. showed in [4] that there is a space $X$ star-determined by convergent sequences while $X$ is not countably compact (strongly 1-star-compact spaces). The space $X$ in Example 2.1 is actually star-determined by convergent sequences. From the proof of Example 2.1, we see that $K_{1}$ is in fact a convergent sequence with the limit point $a$. Since $K_{2}$ is compact, it is of course strongly 1-star-compact and thus there exists a finite subset $F \subset X$ such that $K_{2} \subset S t(F, \mathcal{U})$. Put $S=K_{1} \cup F$; then $S$ is a convergent sequence and $S t(S, \mathcal{U})=X$, which shows that $X$ is star-determined by convergent sequences. Thus we have the following stronger result: there exists a second countable space $X$, star-determined by convergent sequences, which has a $G_{\delta}$-diagonal while $X$ is not metrizable.

Remark 2.3. The subset $\{(1, n) \mid n \in \omega\}$ of the space $X$ in Example 2.1 is a closed subset of $X$, but it is not star-compact (hence not star-determined by convergent sequences). So Example 2.1 also shows that a closed subspace of a star-compact (respectively, star-determined by convergent sequences) space need not be starcompact (respectively star-determined by convergent sequences).

REMARK 2.4. van Douwen et al. [1] showed that countably compact meta-compact spaces are compact and that the space in Example 2.1 is also meta-compact. From Example 2.1, we see that the condition countably compact cannot be replaced by star-compact (star-determined by convergent sequences). That is: there exists a starcompact (star-determined by convergent sequences) meta-compact space which is not compact (not even countably compact).

\section{References}

[1] E. K. van Douwen, G. M. Reed and I. J. Tree, 'Star covering properties', Topology Appl. 39 (1991), 71-103.

[2] G. Gruenhage, 'Generalized metric spaces', in: Handbook of Set-Theoretic Topology (NorthHolland, Amsterdam, 1984), pp. 423-501.

[3] M. Matveev, 'A survey on star covering properties', Topology Atlas, Preprint No. 330, 1998.

[4] J. van Mill, V. V. Tkachuk and R. G. Wilson, 'Classes defined by stars and neighborhood assignments’, Topology Appl. 154 (2007), 2127-2134.

ER-GUANG YANG, Department of Mathematics, Nanjing University,

Nanjing 210093, People's Republic of China

e-mail: egyang@126.com

WEI-XUE SHI, Department of Mathematics, Nanjing University, Nanjing 210093, People's Republic of China

e-mail:wxshi@nju.edu.cn 